\title{
SOLUTION OF SOME TECHNICAL PROBLEMS IN MARKER AND MARKER LOCATOR DEVELOPMENT
}

This paper gives a short description of selected technical problems and their solutions in development of a marker locator. The principle of underground engineering network equipment marking by markers and principle of marker location is explained. Next, mathematical analysis of signal intensity received from an excited marker and its dependence on distance is described. Moreover, influence of over current induced by lightning into a marker is analyzed and practically measured. These facts were used to increase reliability and lifetime of markers.

\section{Introduction}

At present large amount of technical equipment which create engineering networks are hidden under the ground surface, for example telecommunication and power cables, gas and water pipes, sewerage pipes etc. Construction of new underground networks, their repairs, construction of other objects, earthworks etc. require precise marking out of the engineering networks in a given place. Combinations of geodetic and electromagnetic methods are used to determine the position.

One of frequently used electromagnetic methods of the position determining is the marking by markers. Essentially, the marker is a resonant circuit, which is tuned on the concrete frequency different for different marked equipment ( $83 \mathrm{kHz}$ for gas pipes, $101.4 \mathrm{kHz}$ for telecommunication cables, $121.6 \mathrm{kHz}$ for sewerage pipes, 145.7 $\mathrm{kHz}$ for water pipes and $169.8 \mathrm{kHz}$ for power cables). The marker is buried under the ground together with engineering network equipment and marks important points (connection, turning, crossing, point of reparation) or route. The marker can be whenever localized by special device - locator.

\section{Principle of marker - locator system operation}

The marker is resonant circuit connected according to Fig. 1. The locator periodically transmits an excitation signal which excites damped harmonic oscillations in the marker. Frequency of damped oscillations is given by a marker tuning and this signal is received and evaluated by a locator (Fig. 2).

Signals recorded by a digital oscilloscope directly in the locator are depicted in Fig. 3, where trace \#1 shows the excitation current in the locator antenna and trace \#2 shows received response from the marker, i.e. damped harmonic oscillations. The marker is localized by a method which is shown in Fig. 4.

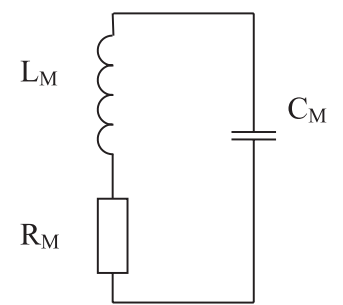

Fig. 1. Connection of marker

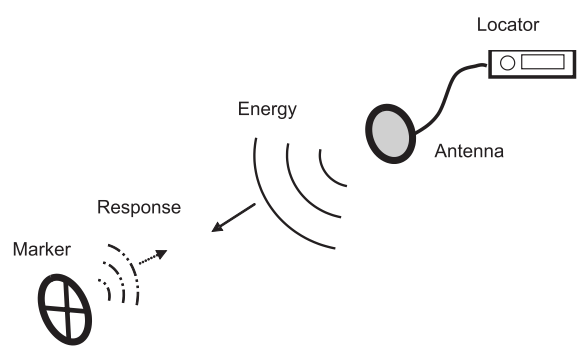

Fig. 2. Principle of marker - locator system operation

\section{Dependence of received signal from marker on distance from locator}

The transmitting antenna of locator is essentially a circular loop coil with radius $r_{L T}$ (with area $S_{L T}$ ) and with $N_{L T}$ turns. The excitation current $I_{L}$ flows by this coil and excites magnetic field. Intensity of the magnetic field in the distance $x$ from the antenna and in its axis direction is given (according to [1]) by formula

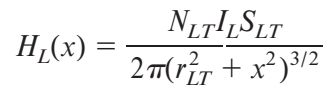

\footnotetext{
* Peter Vestenický

Department of Control and Information Systems, Faculty of Electrical Engineering, University of Žilina. Vel'ký diel, 01026 Žilina, Slovakia. E-mail: peter.vestenicky@fel.utc.sk, Tel.: +421-41-5133345
} 


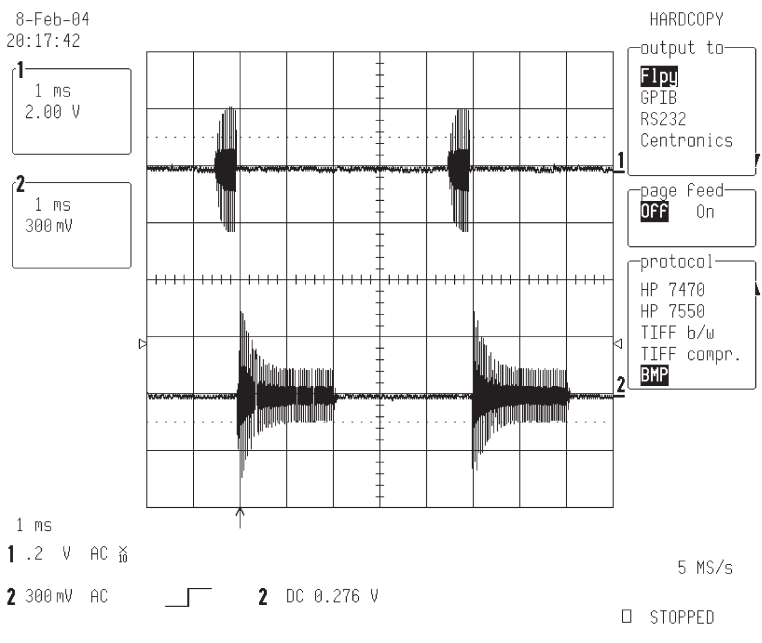

Fig. 3. Excitation and response of marker

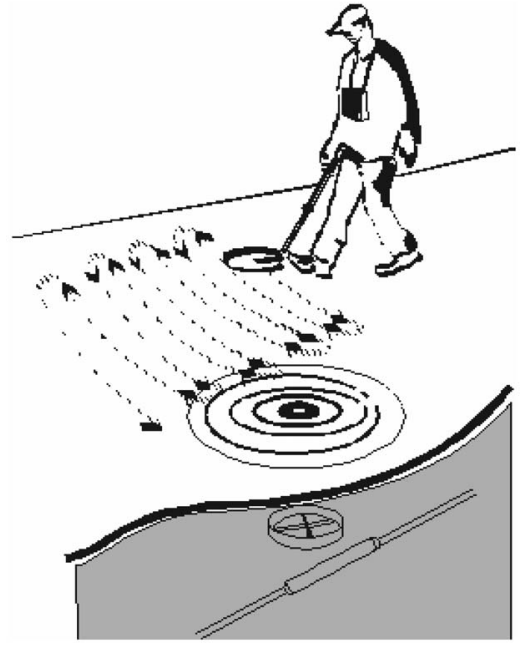

Fig. 4. Principle of marker localization
Assuming that the excitation current is harmonic and has frequency $\omega$ in the marker coil which has $N_{M}$ turns, area $S_{M}$, inductivity $L_{M}$ and its axis is identical with the locator antenna axis the voltage

$$
U_{M}=N_{M} \frac{d \Phi(x)}{d t}=\mu \omega N_{M} S_{M} H_{L}(x)
$$

is induced, where $\Phi(x)$ is magnetic flux and $\mu$ is environment permeability. This voltage causes the current

$$
I_{M}=Q_{M} \frac{U_{M}}{\omega L_{M}}=Q_{M} \frac{\mu N_{M} S_{M} H_{L}(x)}{L_{M}}
$$

in the marker coil, where $Q_{M}$ is the quality factor of marker resonant circuit. The current $I_{M}$ creates magnetic field with intensity $H_{M}$, which in receiving locator antenna place (i.e. again in distance $x$ from marker) induces the voltage $U_{L}$ in receiving locator coil. These magnitudes can be calculated from next formulas:

$$
\begin{aligned}
& H_{M}(x)=\frac{N_{M} I_{M} S_{M}}{2 \pi\left(r_{M}^{2}+x^{2}\right)^{2 / 3}} \\
& U_{L}=\mu \omega N_{L R} S_{L R} Q_{L R} H_{M}(x)
\end{aligned}
$$

or, after combining (1), (3), (4) and (5)

$$
U_{L}=\frac{\mu^{2} \omega N_{L R} N_{M}^{2} N_{L T} Q_{L R} Q_{M} S_{L R} S_{M}^{2} S_{L T} I_{L}}{4 \pi^{2}\left(r_{L T}^{2}+x^{2}\right)^{3 / 2} \cdot\left(r_{M}^{2}+x^{2}\right)^{3 / 2} \cdot L_{M}}
$$

where separate symbols denote:

$N_{L R} \quad$ receiving locator coil number of turns

$S_{L R} \quad$ area of receiving locator coil

$Q_{L R} \quad$ quality factor of locator input tuned circuit.

The marker voltage $U_{M}$ and receiving coil voltage $U_{L}$ calculated from (2) and (6) are graphically depicted in figures 5 and 6 in dependence on distance $x$. For calculation these parameters were used:

- marker parameters

- number of turns $\quad N_{M}=18$
- inductivity

- coil radius

- coil area

- quality factor

$$
L_{M}=169 \mu H
$$$$
r_{M}=0.1 \mathrm{~m}
$$$$
S_{M}=0.0628 \mathrm{~m}^{2}
$$

- transmitting locator coil parameters (shall be chosen subject to [2])

- number of turns

- coil radius

$$
N_{L T}=30
$$

- coil area

- excitation current

$r_{L T}=0.1 \mathrm{~m}$

$S_{L T}=0.0628 \mathrm{~m}^{2}$

- receiving locator coil parameters

- number of turns $\quad N_{L R}=50$

- coil radius

- coil area

- quality factor

- other parameters

- permeability

- frequency

$$
r_{L R}=0.075 \mathrm{~m}
$$$$
S_{L R}=0.03534 \mathrm{~m}^{2}
$$$$
Q_{L R}=15
$$

As (2) depends on (1), it is obvious that the marker induced voltage depends reverse proportionally on the third power of distance $x$. Similarly, it is obvious that voltage on the receiving coil, i.e. induced back from excited marker depends reverse proportionally on the sixth power of distance $x$. Moreover, these simplified calculations do not consider that the amplitude of marker voltage falls exponentially with time (damped harmonic oscillations of marker).

\section{Calculation of voltage induced into marker by current shock wave}

Markers can mark not only non-metallic, but also metallic pipes and cables in an open field. For this reason we must consider the influence of the current waves which spread in a metal conductor on the current and voltage loading of marker components. This shock wave can be created by lightning current or short circuit in power cable. Amplitude of lightning current can achieve up to $50 \mathrm{kA}$ [3]. Considering the ground and metallic conductor 


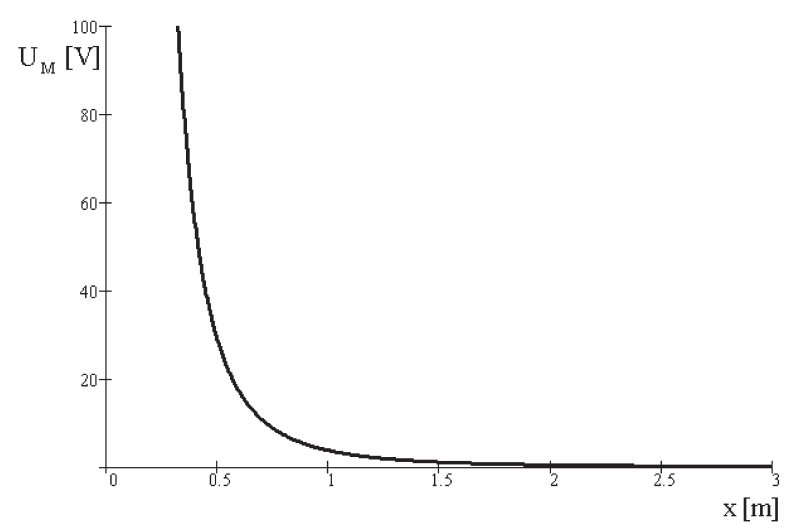

Fig. 5. Voltage on marker

damping factor, we assume about current wave with amplitude $2 \mathrm{kA}$.

Time dependence of such shock wave corresponds approximately with Fig. 7 and can be mathematically modelled by subtraction of two exponentials [3]

$$
i(t)=I\left(e^{-\frac{t}{t_{2}}}-e^{-\frac{t}{t_{1}}}\right)
$$

where $T_{h}$ is front time, $T_{m}$ is half-rear time and $I_{M A X}$ is maximum value of current in the time $T_{h}$. For measuring and calculation the current wave with $T_{h}=8 \mu \mathrm{s}$ and $T_{m}=20 \mu \mathrm{s}$ are being used. Corresponding parameters $t_{1}, t_{2}$ and $I$ must be calculated from a nonlinear equation system

$$
\begin{aligned}
& \frac{t_{1} t_{2}}{t_{2}-t_{1}} \cdot \ln \left(\frac{t_{2}}{t_{1}}\right)=T_{m} \\
& I\left(e^{-\frac{T_{h}}{t_{2}}}-e^{-\frac{T_{h}}{t_{1}}}\right)=I_{M A X} \\
& I\left(e^{-\frac{T_{m}}{t_{2}}}-e^{-\frac{T_{m}}{t_{1}}}\right)=\frac{I_{M A X}}{2}
\end{aligned}
$$

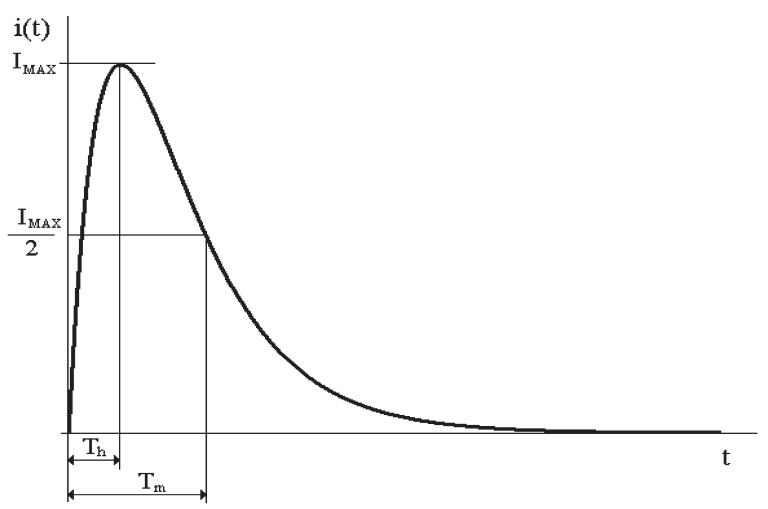

Fig. 7. Shape and parameters of current wave

The numerical solution of the system (8) for given $T_{h}=8 \mu \mathrm{s}$, $T_{m}=21.5 \mu$ s (if $T_{m}=20 \mu$ s the system diverges) and $I_{M A X}=$

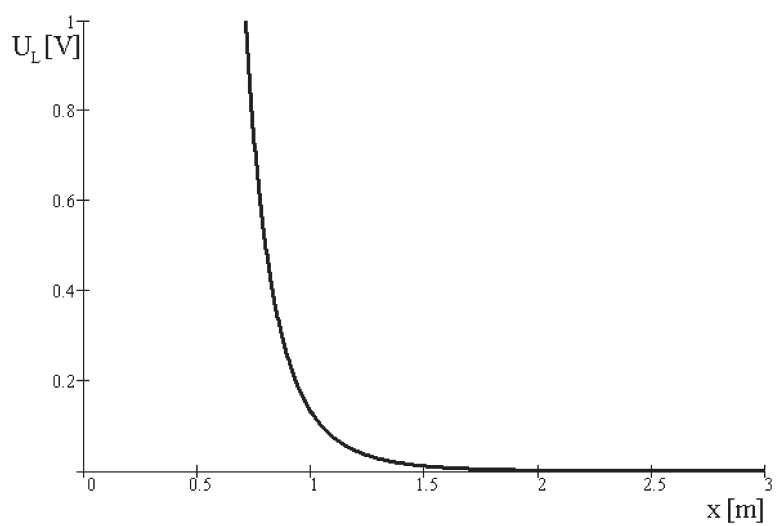

Fig. 6. Voltage on receiving coil

$=2000$ A gives the parameters of shock wave model: $t_{1}=7.18 \mu \mathrm{s}$, $t_{2}=8.95 \mu s$ and $I=24.7 \mathrm{kA}$.

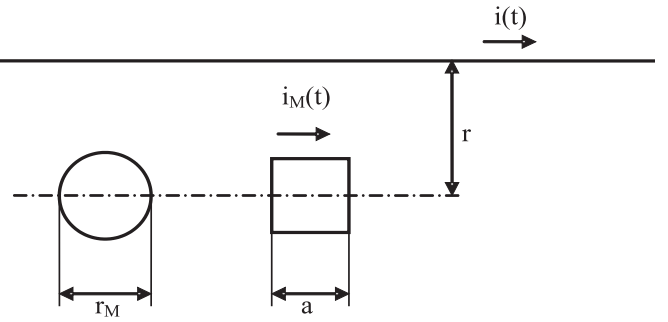

Fig. 8. Arrangement of conductor and marker

We assume that the markers of circular and square shape lay in the distance $r$ from a straight infinitely long conductor by which the current wave $i(t)$ with parameters described above flows (Fig. 8). The current $\mathrm{i}(\mathrm{t})$ creates in marker area magnetic flux

$$
\begin{aligned}
& \Phi(t)=\int_{S_{M}} \mu N_{M} \frac{i(t)}{2 \pi x} d S_{M}=\int_{r-\frac{a}{2}}^{r+\frac{a}{2}} \mu N_{M} \frac{i(t)}{2 \pi x} a \cdot d x= \\
& =\frac{\mu a N_{M}}{2 \pi} \cdot \ln \frac{2 r+a}{2 r-a} i(t)=M i(t)
\end{aligned}
$$

where the circular marker was replaced by a square marker with equivalent area for simplifying the surface integral calculation,

$$
a=\sqrt{\pi} \cdot r_{M}
$$

is length of equivalent square marker side and

$$
M=\frac{\mu a N_{M}}{2 \pi} \cdot \ln \frac{2 r+a}{2 r-a}
$$

is mutual inductivity of a straight conductor and marker coil.

As the marker is essentially resonant circuit, the time dependence of current $i_{M}(t)$ induced into the marker circuit by influence of current wave $i(t)$ (formula (7)) can be calculated from a differential equation 


$$
L_{M} \frac{d^{2} i_{M}(t)}{d t^{2}}+R_{M} \frac{d i_{M}(t)}{d t}+\frac{1}{C_{M}} i_{M}(t)=M \frac{d^{2} i(t)}{d t^{2}}
$$

with respect of initial conditions $i^{\prime}{ }_{M}(0)=0$ and $i_{M}(0)=\frac{M}{L_{M}} I$. $\cdot\left(\frac{1}{t_{1}}-\frac{1}{t_{2}}\right)$. Voltage on the marker capacitor is given by formula

$$
u_{c}(t)=\frac{1}{C_{M}} \int_{0}^{t} i_{M}(\tau) d \tau
$$

The equations (12) and (13) were solved for next parameters:

- marker coil inductivity

- marker coil resistance

$$
L_{M}=1 \mathrm{mH}
$$

- number of turns

$R_{M}=10 \Omega$

- condensator capacity

$N_{M}=54$

- distance of marker from conductor

$$
C_{M}=1.621 \mathrm{nF}
$$

length of equivalent square marker side $a=0.177 \mathrm{~m}$

- mutual inductivity of marker coil and conductor

- current wave $i(t)$ given by formula (7)

with parameters $t_{1}=7.18 \mu \mathrm{s}, t_{2}=8.95 \mu \mathrm{s}, I=24.7 \mathrm{kA}$

and the result of solution is graphically shown in Fig. 9.

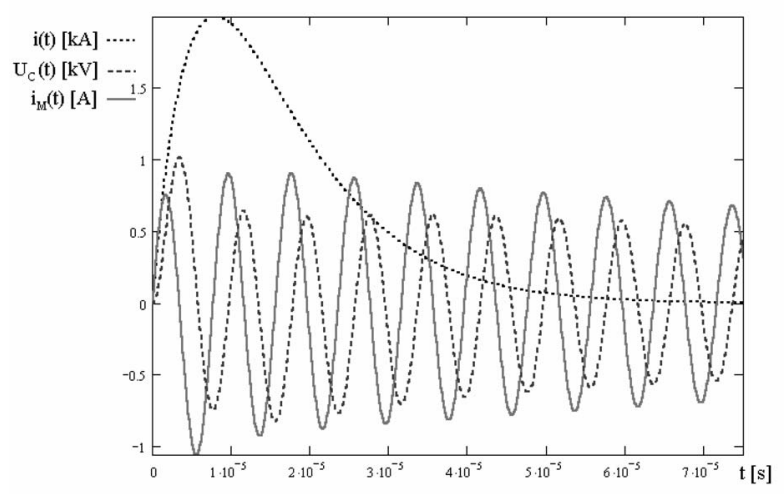

Fig. 9. Time shapes of current wave $i(t)$, marker capacitor voltage $u_{C}(t)$ and marker current $i_{M}(t)$

The current induced in the marker by current wave was experimentally measured with utilization of a pulse generator generating the current pulse with parameters $T_{h}=8 \mu \mathrm{s}, T_{m}=20 \mu \mathrm{s}$, $I_{M A X}=2000 \mathrm{~A}$. The current shape was recorded by a digital oscilloscope as a voltage fall on $0.47 \Omega$ resistor and is in Fig. 10. The

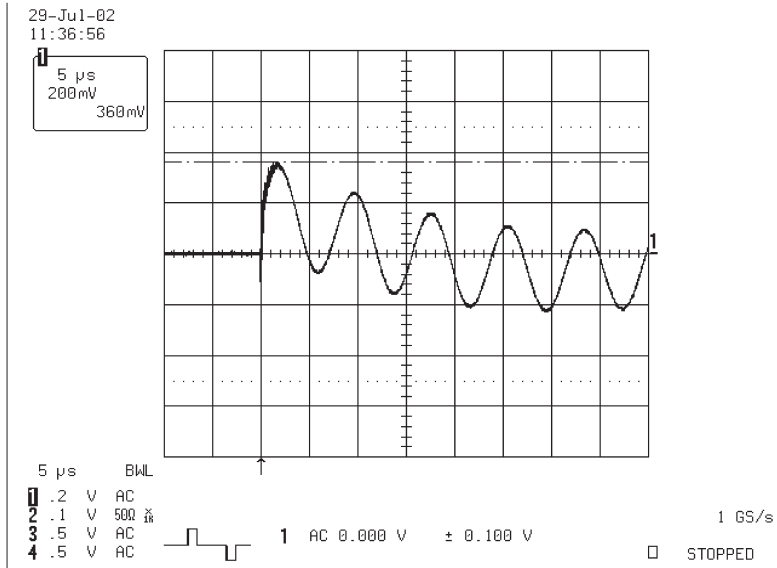

Fig. 10. Really recorded time dependence of marker current represented by voltage on $0.47 \Omega$ resistor

maximum value of current recalculated from voltage $(360 \mathrm{mV})$ and resistance $(0.47 \Omega)$ is $0.76 \mathrm{~A}$.

\section{Conclusion}

The mathematical calculations described in this paper were used in marker locator development, which was realized by the Department of Control and Information systems for the Komplex company as the contract no. EF/28/97.

The calculation of voltage induced from an excited marker back to the receiving antenna showed that this voltage is reverse proportionally dependent on the sixth power of distance. This fact limits practically achievable distance from which the marker can be localized to the value of 2.5 , maximum $3 \mathrm{~m}$.

The calculation and measuring of voltage and current in the marker induced from current wave flowing by a metal conductor of marked equipment showed that these influences cannot be neglected. For increasing the marker reliability and lifetime (marker lifetime must be greater than 40 years) the marker capacitor must be high voltage and current type. Therefore the capacitors with working voltage of $1500 \mathrm{~V}$ are used. Similarly, insulation of the marker coil conductor must be carefully chosen.

At present the development of a new marker type based on RFID (Radio Frequency Identification) technology is in progress. The RFID technology enables to supplement new function of marker - identification of underground device by a numeric code.

\section{References}

[1] GEHRING, U., ROZ, TH.: RFID made easy. EM Microelectronic-Marin SA. Marin, Switzerland, 2000

[2] ETSI EN 300330-1 (V1.3.2) Electromagnetic compatibility and Radio spectrum Matters (ERM); Short Range Devices (SRD); Radio equipment in the frequency range $9 \mathrm{kHz}$ to $25 \mathrm{MHz}$ and inductive loop systems in the frequency range $9 \mathrm{kHz}$ to $30 \mathrm{MHz}$; Part 1: Technical characteristics and test methods

[3] ROUS, Z.: Overvoltage Protection of telecommunication lines and equipment (in Czech). NADAS, Praha, 1981 